\title{
Anidación y conservación de la tortuga lora (Lepidochelys olivacea) en playa Drake, península de Osa, Costa Rica (2006 a 2012)
}

\author{
Robert James $^{1}$ \& David Melero ${ }^{2}$ \\ 1. Fundación Corcovado Lon Willing Ramsey Jr. Moravia, Los Colegios Norte, San José, Costa Rica, P.O. Box 191- \\ 1007; robert@corcovadofoundation.org \\ 2. Iniciativa Carey del Pacífico Oriental, 2965 Redwood Street, San Diego, CA 92104, USA; dmelero@hawksbill.org
}

Recibido 11-VIII-2014. Corregido 20-XI-2014. Aceptado 22-XII-2014.

\begin{abstract}
Nesting and conservation of the Olive Ridley sea turtle (Lepidochelys olivacea) in playa Drake, Osa Peninsula, Costa Rica (2006-2012). The nesting of the Olive Ridley (Lepidochelys olivacea) sea turtle was studied from 2006 to 2012 in Drake Bay, Costa Rica, an important solitary nesting site and center of eco-tourism in the Osa Peninsula. During this period, 958 nests were recorded (mean: 136.9 nests per season; density: 3.8 nests $/ 100 \mathrm{~m}$ of beach per season), of which $38 \%$ were relocated to a hatchery. The incidence of poaching was reduced from $85 \%$ in 2005 to a mean of $10.1 \%$ from 2006-2012. A total of 335 nesting females were tagged; the mean curved length of carapace was $66.1 \mathrm{~cm}$, the mean curved width was $70.2 \mathrm{~cm}$, and the mean number of eggs per nest was 96.3. A mean rate of reproductive success of $79.2 \%$ was obtained and over 61000 hatchlings were liberated from the hatchery. This project is an example of a successful community-based conservation and eco-tourism initiative. Rev. Biol. Trop. 63 (Suppl. 1): 117-129. Epub 2015 April 01.
\end{abstract}

Key words: Olive Ridley, Cheloniidae, nesting biology, sea turtle, poaching, community-based conservation.

Existen cuatro especies de tortugas marinas que anidan en el Pacífico costarricense: lora (Lepidochelys olivacea), baula (Dermochelys coriacea), verde (Chelonia mydas) y carey (Eretmochelys imbricata). Todas ellas se encuentran amenazadas y una de ellas incluso en peligro crítico de extinción (Seminoff, 2004; Abreu-Grobois \& Plotkin, 2008; Mortimer \& Donnelly, 2008; Wallace, Tiwari, \& Girondot, 2013). Históricamente muchas comunidades nativas de alrededor del mundo se han beneficiado de la carne, caparazón, huevos y aceite de las tortugas (Frazier, 2003). A pesar de haber existido esta captura histórica de tortugas marinas carente de regulación durante siglos, no es hasta mediados del siglo XX cuando aparecen nuevas amenazas que hacen que las poblaciones de tortugas marinas disminuyan drásticamente (Jackson et al., 2001). Entre ellas se incluyen: el tráfico comercial a gran escala, la pesca incidental, al igual que la constante pérdida o degradación de los hábitats costeros (Spotila, Reina, Steyermark, Plotkin, \& Paladino, 2000; Jackson et al., 2001).

De las cuatro especies presentes en el Pacífico de Costa Rica, todavía todas ellas anidan en la península de Osa (Drake, 1996; Bernal Castro, Sánchez Mendoza, Cheetham, \& Herrera, Vargas, 2013) y potencialmente podrían hacerlo de nuevo en bahía Drake (James et al., 2011; James et al., 2013). La anidación de la tortuga lora (Lepidochelys olivacea) es un fenómeno común en muchas playas del Pacífico de Costa Rica entre los meses de julio y diciembre (Cornelius \& Robinson, 1985; Cornelius, Ulloa, \& Robinson, 1991; Drake, 1996; Valverde, Cornelius, \& Mo, 1998; Brenes Arias, 2012; Viejobueno, Adams, \& Arauz, 2012; Bernal Castro et al., 2013). La península de Osa posee las playas de anidación solitaria más importantes 
del país para esta especie (Drake, 1996; Bernal Castro et al., 2013). Bahía Drake es un claro ejemplo de la dinámica que han mostrado otras playas de anidación por todo el mundo. Estudios preliminares en playa Drake han mostrado un bajo número de eventos de anidación, lo cual contrasta con estimaciones obtenidas a partir de entrevistas con pobladores locales que citan anidaciones muy superiores apenas tres o cuatro décadas atrás (datos no publicados). Todos estos indicios señalan que la tortuga lora en bahía Drake al inicio de este proyecto de conservación conformaba una población en paulatino descenso con un saqueo superior al 85\% (Sánchez, Melero, Alonso, \& Bigler, 2006). Aunque la tortuga lora es considerada como la más abundante de las siete especies de tortugas marinas reconocidas a nivel mundial (Dutton, Davis, Guerra, \& Owens, 1996; Karl \& Bowen, 1999), se cree que la población actual es sólo un remanente de lo que alguna vez fue. Desafortunadamente, la categoría de protección brindada por la UICN (Unión Mundial para la Conservación de la Naturaleza) a esta especie fue devaluada, pasando su estado de conservación de especie "En Peligro" a especie "Vulnerable" (Abreu-Grobois \& Plotkin, 2008). Si bien se trata de una especie que anida por cientos de miles en unas pocas playas del mundo como Ostional o Nancite en Costa Rica, debe señalarse que el éxito de eclosión en estas áreas se encuentra por debajo del 5\% (Cornelius et al., 1991; Pritchard, 2007). En contraste, en las playas de anidación solitaria el éxito reproductivo puede ser considerablemente más alto, incluso superior al $80 \%$ (Sánchez et al., 2006; Melero, Le-Garec, Andreu, Ramírez, \& Moreno, 2009; James et al., 2012; Bernal Castro et al., 2013), un hecho que destaca la importante contribución de la anidación solitaria para el mantenimiento de la población de la tortuga lora en el Pacífico Oriental.

En 2006 se inician los esfuerzos de conservación de tortugas marinas en la comunidad de El Progreso de mano de Fundación Corcovado en respuesta a denuncias locales que alertaban de una disminución drástica del número de nidos año tras año. Durante los siete años que cubre esta publicación, este monitoreo se produjo ininterrumpidamente logrando un sólido y creciente apoyo local además de una reducción drástica del número de nidadas saqueadas (Melero, Alonso, Rubiano, \& Careaga, 2008; Melero et al., 2010). El desarrollo de un programa de conservación basado en comunidades locales ha sido identificado en diversos lugares como un modelo de gran éxito en proyectos de tortugas marinas cuando existe una integración de actores locales en la toma de decisiones del propio programa. Es en esta línea que se sientan las bases de un modelo de este tipo en El Progreso con el entrenamiento de diversas personas locales en las actividades de monitoreo de campo durante los primeros años de proyecto hasta impulsar la formación de ACOTPRO (Asociación Comunitaria para la Conservación de la Tortuga Marina de El Progreso) en 2009 (Le-Garec, Melero, \& Andreu, 2010).

El objetivo de este documento es destacar los éxitos de conservación logrados dentro del proyecto de tortugas marinas impulsado de manera conjunta por Fundación Corcovado y la comunidad local de El Progreso. Se quiere ahondar en el concepto de proyecto de conservación de tortugas marinas basado en comunidades y sus innumerables beneficios socio-económicos así como ambientales a largo plazo.

\section{MATERIALES Y MÉTODOS}

Playa Drake se encuentra situada entre la desembocadura del río Drake al sur ( $8^{\circ} 41^{\prime} 59.0^{\prime \prime}$ $\left.\mathrm{N}-83^{\circ} 39^{\prime} 11.9^{\prime \prime} \mathrm{W}\right)$ y Punta Ganadito al norte $\left(8^{\circ} 43^{\prime} 53.3^{\prime \prime} \mathrm{N}-83^{\circ} 38^{\prime} 57.5^{\prime \prime} \mathrm{W}\right)$. A efectos de manejo, playa Drake posee dos sectores bien definidos: el sector norte de aproximadamente $1.5 \mathrm{~km}$, con una amplitud de playa mayor y una primera línea de vegetación más boscosa, y el sector sur de aproximadamente $2,1 \mathrm{~km}$, que comprende una franja de playa estrecha y con mayor pendiente tras la cual circula de forma paralela el río Drake. Ambos están divididos por una barra arenosa que se abre periódicamente casi cada año durante la época de lluvias. Si no es de forma natural, es ayudada por 
la mano del hombre para que ingresen varias especies marinas a la laguna, además de mantener el nivel del agua y así evitar inundaciones.

La temporada de anidación de tortuga lora suele comenzar en julio y extenderse hasta diciembre en playas de anidación solitaria. Sin embargo, ésta puede adelantarse o retrasarse y extenderse o reducirse ligeramente en playas de mayor o menor número de nidos respectivamente. El trabajo de monitoreo de playa y reubicación de nidos se ha realizado ininterrumpidamente desde 2006, iniciándose en la primera semana de agosto (2006-2007) o la segunda semana de julio (2008-2012), y extendiéndose hasta la segunda semana de diciembre. Todas las noches se realizaron cuatro patrullas de cuatro horas cubriendo cada uno de los sectores entre las 20:00h y las 04:00h para observar y anotar todo lo pertinente a la anidación de las tortugas, y reubicar nidos al vivero.

El personal local, como miembros de la asociación ACOTPRO, fue entrenado para tomar datos científicos y proteger adecuadamente nidadas de tortugas marinas en el proyecto de conservación de Playa Drake. El número de personas locales entrenadas ascendió progresivamente hasta 50 personas en el periodo señalado en este documento. Como estrategia de sostenibilidad financiera del propio proyecto de conservación así como de fortalecimiento del esfuerzo de monitoreo en playa, voluntarios internacionales fueron reclutados. Estos participantes llegaron como donantes activos del proyecto poniendo sus manos en la conservación de la tortuga marina y dejando un beneficio económico al proyecto reinvertido en su implementación a medio plazo.

Los datos científicos recogidos en las hembras anidantes fueron tomados de acuerdo con la metodología de Cornelius et al. (1991), y Pritchard et al. (1983), incluyendo datos biométricos como la longitud curva del caparazón (LCC), el ancho curvo del caparazón (ACC), número de huevos (para los nidos reubicados) y la profundidad del nido (desde 2007); datos referentes al comportamiento anidatorio (zona de playa donde anida, la orientación de la tortuga desovando, distribución espacial y temporal de la tortuga que anida) así como otras características para identificar al animal (marcas metálicas, presencia de epibiontes, y posibles daños). Según el manejo realizado con la nidada, se clasificaron como nido reubicado en playa, nido reubicado en vivero, nido natural o in situ, nido perdido por saqueo o nido depredado. Independientemente del destino de las nidadas, cada evidencia de anidación fue camuflada para desalentar a los recolectores ilegales.

Para cada salida de tortuga a playa se calculó el número de horas desde la última marea alta. Con este dato, y asumiendo que un ciclo completo de marea tiene una duración de $12.43 \mathrm{~h}$, se asignó cada salida a un grupo de marea: media marea decreciente (1.55-4.66h), marea baja (4.67-7.77h), media marea creciente $(7.78-10.88 \mathrm{~h})$ y marea alta (10.89-12.43h y $0-1.54 \mathrm{~h})$.

Las tortugas se marcaron al finalizar la ovoposición o durante la fase final del desove donde las tortugas loras están en trance e inmóviles. Se marcó cada tortuga con dos placas metálicas colocadas en el borde interior de las aletas anteriores, preferentemente entre la primera y la segunda escama. Las marcas que se utilizaron fueron Inconel \#681 de la National Band \& Tag Company (NBTC). Las series utilizadas fueron OSA0901-OSA0950 (2006 a 2007), OSA1401-OSA1500 (2007 a 2008), OSA2201-OSA2300 (2008), NG0201NG0350 (2009), OP0001-OP0200 (2010 a 2011), OSA02401-OSA02500 (2011), OP0401OP0500 (2011 a 2012), OP1701-OP1800 (2011 a 2012), OP1801-OP1900 (2012), OP1501OP1600 (2012), y OP2401-OP2500 (2012).

El vivero fue montado y desmontado cada temporada alternando su localización entre dos sitios próximos a una torre de vigilancia localizada en el sector sur de playa Drake. Seguido al desbrozado del área y tamizado de la arena, se levantó la estructura de madera y malla plástica para completar el recinto. Cada nido ocupó un área de $1 \mathrm{~m}^{2}$, y el ordenamiento de las nidadas se estableció mediante carteles con códigos. El orden de llenado fue aleatorio registrándose únicamente en una bitácora en vivero. La reubicación de las nidadas en el vivero se realizó 
cuidadosa y rápidamente; el diámetro (desde 2007) y peso de muestras de 10 huevos de cada nido fueron medidos, y los nidos fueron relocalizados a la misma profundidad a la que se encontraron en playa (promedio: $43 \mathrm{~cm}$ ). Se realizaron cuatro turnos de vigilancia en vivero de seis horas cada uno iniciando a las 06:00h, 12:00h, 18:00h y 00:00h, de forma que se alcanzaran $24 \mathrm{~h}$ de vigilancia por día durante toda la temporada de anidación.

Se tomaron datos biométricos de 10 neonatos para cada uno de los nidos eclosionados en vivero. Además de pesarlos mediante una balanza de resorte (pesola ${ }^{\circledR} 100 \mathrm{gr}$ ), se anotaron medidas del caparazón tomando tanto el curvo (LCC, ACC) con cinta métrica, como el recto (LRC, ARC) mediante pie de rey. Los neonatos se liberaron inmediatamente cerca del vivero, pero nunca en el mismo lugar. Se determinaron los éxitos de eclosión y emergencia y se realizaron exhumaciones para cada nido $48 \mathrm{~h}$ después de la emergencia del primer neonato de acuerdo con Crastz (1982). Para cada nido excavado se determinó el número de cáscaras vacías, huevos abiertos con embrión a medio salir (a término), depredados, neonatos vivos y muertos, huevos sin embrión, huevos sin eclosionar con embrión señalando su fase de desarrollo (I, II, III o IV), gemelos, albinos, y/o deformidades.

En las temporadas de anidación 20112012, la temperatura de la arena fue registrada en varios puntos de la playa y dentro del vivero, a una profundidad de $43 \mathrm{~cm}$, con registradores automáticos (dataloggers) (HOBO Pendant Temperature UA-001-08/64 [precisión $\left.\pm 0.47^{\circ} \mathrm{C}\right]$ ). La proporción de los sexos de todos los nidos que nacieron en el vivero se calculó utilizando el método indirecto, basado en la temperatura media durante el periodo termo-sensible al final del primer tercio del periodo de incubación (Mrosovsky \& Yntema, 1980; Spotila, Standora, Morreale, \& Ruiz, 1987; Merchant-Larios, Ruiz-Ramírez, Moreno-Mendoza, \& Marmolejo-Valencia, 1997; Wibbels, 2003). La temperatura pivote ha sido reportado como $30.5^{\circ} \mathrm{C}$ para la tortuga lora, y se obtiene $100 \%$ machos a los $27.0^{\circ} \mathrm{C}$ y $100 \%$ hembras a los $32.0^{\circ} \mathrm{C}$ (McCoy, Vogt, \& Censky, 1983; Wibbels, Rostal, \& Byles, 1998). Una función lineal entre 27.0 y $30.5^{\circ} \mathrm{C}$, y entre 30.5 y $32.0^{\circ} \mathrm{C}$ se utilizó para estimar la proporción de sexos de los nidos del vivero, utilizando la temperatura media de la arena al final del primer tercio de incubación para cada nido.

\section{RESULTADOS}

Entre julio de 2006 y diciembre de 2012, un total de 958 nidos de tortuga lora fueron registrados en playa Drake (Fig. 1) de los cuales $363(37.9 \%)$ fueron reubicados al vivero. Se identificaron un total de 335 hembras con placas metálicas. El promedio anual de nidos fue de 136.9 con una densidad de 3.80 nidos/100m de playa, y un promedio de 0.81 tortugas por noche y temporada. La temporada de anidación más exitosa fue la de 2008 con 177 nidos, y la menos exitosa fue la de 2007 con tan sólo 98 nidos.

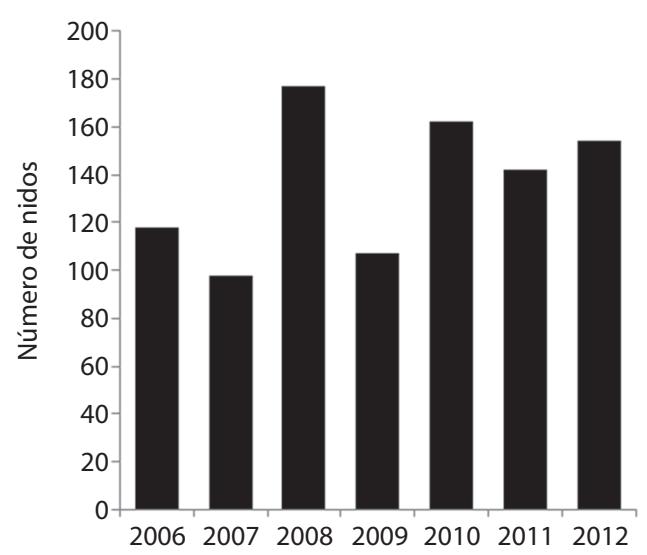

Fig. 1. Número de nidos totales de L. olivacea por año en playa Drake, península de Osa, durante las temporadas 2006-2012.

Fig. 1. Distribution of the total nests laid by L. olivacea each season from 2006 to 2012.

Un análisis comparativo de la distribución temporal de la anidación para cada temporada entre 2006 y 2012 (Fig. 2), ha permitido señalar a los meses de septiembre y agosto como los que presentan el mayor número de anidaciones, 


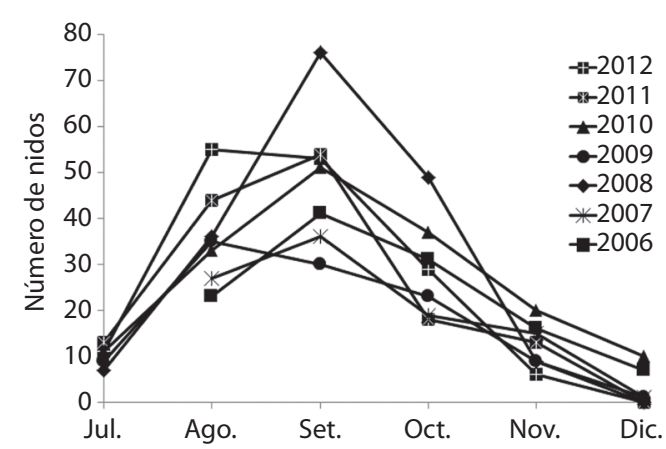

Fig. 2. Frecuencia de anidación mensual de las hembras de L. olivacea para las temporadas de 2006 a 2012 . $†$ No hay datos para julio de 2006 ni 2007, porque el proyecto empezó el primero de agosto en estos años.

Fig. 2. Monthly nesting frequency for $L$. olivacea for each season from 2006 to 2012. †There are no data for July 2006 or 2007, because the project began in August during these first two years. con un $35.6 \%$ y un $26.4 \%$ respectivamente. Asimismo, se observa un cambio gradual en los meses de anidación durante los últimos siete años. La cantidad de nidos puestos es mayor en los meses de julio, agosto y septiembre, mientras que disminuyen en los meses de octubre, noviembre y diciembre.

La mayor intensidad de anidación se dio entre los mojones 15 y 20 (Fig. 3). De los 958 nidos, el 23.8\% (Desviación Estándar, D.E. $=12.1 \%$ ) se encontraron debajo de la línea de marea alta (Zona 1), 64.7\% (D.E. $=10.7 \%$ ) entre la línea de marea alta y la vegetación (Zona 2 ), y $11.5 \%$ (D.E. $=8.4 \%$ ) en la vegetación (Zona 3).

En 2007, el $2.9 \%$ de las tortugas que llegaron a desovar traían placas metálicas. Esta proporción aumentó al $11.1 \%$ en 2008 ,
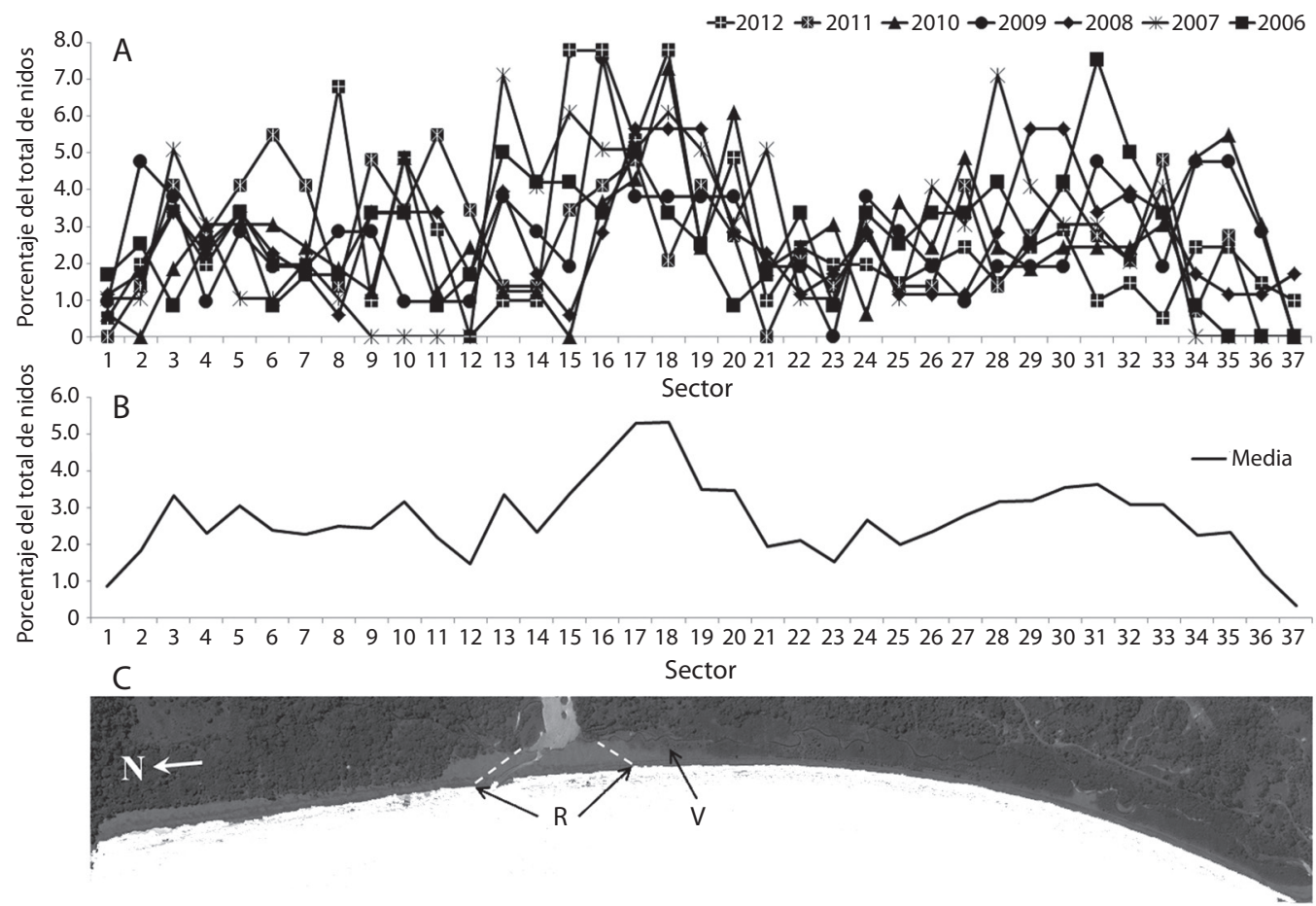

Fig. 3. A. Distribución espacial de los nidos de L. olivacea en los 37 sectores de la playa Drake, península de Osa, trazada como el porcentaje del total de nidos encontrados por sector, de 2006 a 2012. B. Promedia distribución espacial. C. Mapa de la playa. R. Zona de la desembocadura del río, V. Sitio del vivero, N=Norte.

Fig. 3. A. Spatial distribution of L. olivacea nests across 37 sectors of Drake Beach, shown as percentage of total nests found in each sector during each season from 2006 to 2012, B. Mean spatial distribution. C. Map of Drake Beach. R. Zone of river mouth, V. Hatchery site, $\mathrm{N}=$ North. 
al $18.8 \%$ en 2009 , y al $30.6 \%$ en 2010 . Desde 2010 , esta proporción descendió al $17.4 \%$ en 2011, y al $10.3 \%$ en 2012. Cada temporada, un promedio del $67.6 \%$ (D.E. $=4.79 \%$ ) de las tortugas emergieron durante la primera patrulla (20:00-00:00h). El 54.8\% (D.E.=1.86\%) emergieron a media marea, el 33.4\% (D.E. $=1.66 \%$ ) en marea baja, y el $11.7 \%$ (D.E. $=2.54 \%$ ) durante la marea alta.

Se realizaron análisis comparativos entre la frecuencia de anidación y las fases lunares indicando que la mayor intensidad de anidación ocurre 3-5 días después de la luna nueva (días 4-6 del ciclo lunar), y 3-4 días después de la luna llena (días 19-20 del ciclo lunar) (Fig. 4). Estos dos períodos corresponden a las dos mareas vivas del ciclo lunar, y se caracterizan por la marea baja o marea decreciente durante la primera patrulla.

Según los datos mostrados en el cuadro 1, el promedio de la longitud curva del caparazón (LCC) de 380 tortugas muestreadas fue de $66.1 \mathrm{~cm}(\mathrm{D} . \mathrm{E} .=2.96 \mathrm{~cm})$, mientras que el promedio del ancho curvo del caparazón (ACC) fue de $70.2 \mathrm{~cm}$ (D.E. $=2.88 \mathrm{~cm}$ ). El número promedio de huevos de 644 nidos muestreados fue de 96.3 (D.E.=21.0), y el promedio para la profundidad de 451 nidos muestreados fue de $42.6 \mathrm{~cm}$ (D.E. $=4.58 \mathrm{~cm})$.

Entre 2006 y 2012, un total de 363 nidos fueron reubicados en el vivero, con un periodo promedio de incubación de 57.7 días (D.E. $=4.63$ días; rango=45-74 días). Fueron liberados al mar más de 61000 neonatos. La temperatura promedio de la arena en el vivero se mantuvo en $\operatorname{los} 29.0^{\circ} \mathrm{C}$ (D.E. $=1.69^{\circ} \mathrm{C}$; rango $=25.7$ $32.1^{\circ} \mathrm{C}$ ) en 2011 , y en $30.3^{\circ} \mathrm{C}$ (D.E. $=0.91^{\circ} \mathrm{C}$; ámbito $=28.1-31.1^{\circ} \mathrm{C}$ ) en 2012 . El $71.4 \%$ de los nidos tuvieron un sesgo masculino en 2011 y el $66.1 \%$ de los nidos en 2012. Adicionalmente, la proporción de sexos estimada para los neonatos liberados del vivero fue $56 \%$ machos y 44\% hembras en 2011, mientras que para 2012 correspondió a $52 \%$ machos y $48 \%$ hembras.

A como puede verse en el cuadro 2, el éxito promedio de eclosión para los nidos reubicados en vivero entre 2006 y 2012 , fue de $79.2 \%$ (D.E. $=9.32 \%$ ), mientras que el de emergencia fue de $76.6 \%$ (D.E. $=8.89 \%$ ). Para los nidos reubicados a la playa, la tasa promedio de eclosión

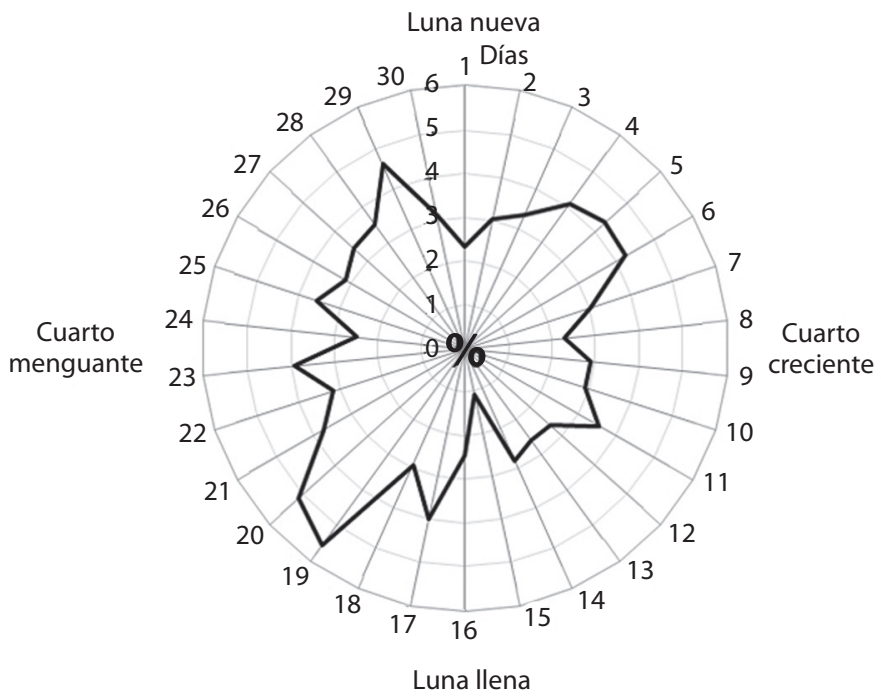

Fig. 4. Relación entre la frecuencia de anidación de las hembras de L. olivacea y el ciclo lunar en playa Drake, península de Osa, trazada como el porcentaje del total de desoves durante cada temporada, de 2006 a 2012.

Fig. 4. Relation between nesting frequency of L. olivacea and lunar cycle, shown as percentage of total nests laid during each season from 2006 to 2012 . 
CUADRO 1

Valores mínimo y máximo, media y desviación estándar del longitud curva del caparazón (LCC), ancho curvo del caparazón (ACC), número de huevos y profundidad de nido de las hembras anidantes de L. olivacea por año, en playa Drake, península de Osa, en las temporadas de anidación 2006-2012

TABLE 1

Minimum and maximum values, mean and standard deviation of the curved carapace length (LCC) and width (ACC), number of eggs, and nest depth for L. olivacea turtles and nests on Drake Beach during each nesting season from 2006 to 2012

\begin{tabular}{|c|c|c|c|c|c|}
\hline & Valor mínimo & Valor máximo & Media & D.E. & $\mathrm{n}$ \\
\hline \multicolumn{6}{|l|}{ Temporada 2006} \\
\hline $\mathrm{LCC}(\mathrm{cm})$ & 58.5 & 71.1 & 65.7 & 3.22 & 26 \\
\hline $\mathrm{ACC}(\mathrm{cm})$ & 66.5 & 76.0 & 70.3 & 2.60 & 26 \\
\hline № de huevos & 54 & 136 & 100 & 18.1 & 65 \\
\hline \multicolumn{6}{|l|}{ Temporada 2007} \\
\hline $\mathrm{LCC}(\mathrm{cm})$ & 60.5 & 72.5 & 67.0 & 3.00 & 32 \\
\hline $\mathrm{ACC}(\mathrm{cm})$ & 66.5 & 78.6 & 71.1 & 3.20 & 31 \\
\hline № de huevos & 7 & 133 & 94.1 & 27.0 & 53 \\
\hline Prof. de nido $(\mathrm{cm})$ & 34.5 & 57.0 & 42.1 & 5.20 & 39 \\
\hline \multicolumn{6}{|l|}{ Temporada 2008} \\
\hline $\operatorname{LCC}(\mathrm{cm})$ & 59.5 & 72.5 & 66.2 & 2.83 & 51 \\
\hline $\mathrm{ACC}(\mathrm{cm})$ & 64.0 & 77.5 & 70.1 & 2.78 & 51 \\
\hline № de huevos & 24 & 156 & 95.7 & 19.8 & 104 \\
\hline Prof. de nido $(\mathrm{cm})$ & 30.0 & 51.0 & 40.2 & 4.27 & 25 \\
\hline \multicolumn{6}{|l|}{ Temporada 2009} \\
\hline $\mathrm{LCC}(\mathrm{cm})$ & 61.0 & 67.5 & 67.5 & 2.01 & 34 \\
\hline $\mathrm{ACC}(\mathrm{cm})$ & 65.0 & 76.0 & 71.1 & 3.04 & 34 \\
\hline № de huevos & 32 & 128 & 98.5 & 19.5 & 60 \\
\hline Prof. de nido $(\mathrm{cm})$ & 22.0 & 54.0 & 41.6 & 5.96 & 52 \\
\hline \multicolumn{6}{|l|}{ Temporada 2010} \\
\hline $\operatorname{LCC}(\mathrm{cm})$ & 60.0 & 77.0 & 66.1 & 3.11 & 83 \\
\hline $\mathrm{ACC}(\mathrm{cm})$ & 62.0 & 78.0 & 69.8 & 3.21 & 82 \\
\hline № de huevos & 29 & 135 & 95.9 & 21.3 & 128 \\
\hline Prof. de nido $(\mathrm{cm})$ & 32.0 & 66.0 & 43.9 & 4.31 & 113 \\
\hline \multicolumn{6}{|l|}{ Temporada 2011} \\
\hline $\mathrm{LCC}(\mathrm{cm})$ & 58.0 & 72.0 & 66.0 & 2.67 & 84 \\
\hline $\mathrm{ACC}(\mathrm{cm})$ & 64.5 & 76.0 & 70.0 & 2.64 & 84 \\
\hline № de huevos & 40 & 138 & 96.3 & 19.9 & 115 \\
\hline Prof. de nido $(\mathrm{cm})$ & 33.0 & 50.0 & 42.2 & 3.94 & 104 \\
\hline \multicolumn{6}{|l|}{ Temporada 2012} \\
\hline $\operatorname{LCC}(\mathrm{cm})$ & 61.0 & 78.0 & 65.4 & 3.00 & 72 \\
\hline $\mathrm{ACC}(\mathrm{cm})$ & 63.0 & 75.0 & 69.5 & 2.68 & 72 \\
\hline № de huevos & 12 & 136 & 96.4 & 21.7 & 119 \\
\hline Prof. de nido $(\mathrm{cm})$ & 29.0 & 54.0 & 42.7 & 4.39 & 118 \\
\hline
\end{tabular}

fue de $78.5 \%$ (D.E. $=5.07 \%$ ), mientras que el de emergencia fue de $70.6 \%$ (D.E. $=7.58 \%$ ).

Los datos biométricos de huevos y neonatos son expresados en el cuadro 3. El diámetro promedio de los huevos (2007 a 2012) fue $3.63 \mathrm{~cm}$ (D.E. $=0.13 \mathrm{~cm}$ ), mientras que el peso promedio (2006 a 2012) fue $32.2 \mathrm{~g}$ (D.E. $=2.60 \mathrm{~g}$ ). El promedio de la longitud curva del caparazón (LCC) registrado para los neonatos (2006 a 2012) fue de $4.38 \mathrm{~cm}$ (D.E. $=0.18 \mathrm{~cm}$ ); el ancho curvo del caparazón
(ACC) fue de $4.54 \mathrm{~cm}$ (D.E. $=0.55 \mathrm{~cm}$ ), y el del peso fue $17.7 \mathrm{~g}$ (D.E. $=1.84 \mathrm{~g})$.

Desde 2006 el porcentaje del saqueo de nidos se mantuvo en un promedio del $10.1 \%$ (D.E. $=2.89 \%$ ) (Fig. 5).

\section{DISCUSIÓN}

La disminución de las poblaciones de tortugas marinas reproductoras en playas como las 
CUADRO 2

Éxito de eclosión y de emergencia de los nidos de L. olivacea reubicados en playa y en vivero por año en playa Drake, en las temporadas 2006-2012

TABLE 2

Mean rate of eclosion and emergence for L. olivacea nests analyzed relocated on the beach or to the hatchery during each nesting season on Drake Beach from 2006 to 2012

\begin{tabular}{lccc} 
& $\begin{array}{c}\text { Media } \\
\text { eclosión \% }\end{array}$ & $\begin{array}{c}\text { Media } \\
\text { emergencia \% }\end{array}$ & $\mathrm{n}$ \\
$\begin{array}{l}\text { Temporada 2006 } \\
\text { Playa }\end{array}$ & 81.8 & 75.8 & $\mathrm{n} . \mathrm{i}$ \\
Vivero & 89.2 & 85.2 & 44 \\
Temporada 2007 & & & \\
Playa & 75.4 & 68.1 & n.i. \\
Vivero & 85.8 & 83.1 & 33 \\
Temporada 2008 & & & \\
Playa & 76.7 & 73.0 & 48 \\
Vivero & 62.6 & 61.4 & 48 \\
Temporada 2009 & & & \\
Playa & 81.6 & 77.3 & 24 \\
Vivero & 75.2 & 73.3 & 40 \\
Temporada 2010 & & & \\
Playa & 76.2 & 73.9 & 11 \\
Vivero & 80.4 & 78.4 & 92 \\
Temporada 2011 & & & \\
Playa & 71.2 & 54.8 & 6 \\
Vivero & 74.2 & 70.0 & 106 \\
Temporada 2012 & & & \\
Playa & 86.4 & 71.1 & 7 \\
Vivero & 86.9 & 84.8 & 110 \\
\hline
\end{tabular}

n.i.: No hay información; datos fueron perdidos. n.i.: No information available; data were lost.

de bahía Drake es un reflejo de la situación que afrontan estos reptiles a nivel global (Wallace, et al., 2011). Históricamente, los pobladores locales conocieron la presencia de cuatro especies diferentes de tortugas marinas que llegaban a desovar a playa Drake (com. pers.). Lamentablemente en la actualidad sólo la tortuga lora llega constantemente, con unos aislados eventos reproductivos de tortugas carey y verde (Melero et al., 2009). Estudios previos a 2006, describían una pérdida anual de nidos superior al $85 \%$ debido al saqueo en playa Drake (Sánchez et al., 2006). Siete años después de trabajo ininterrumpido podemos vislumbrar esperanzas de recuperación para esta población con una protección del $90 \%$ de las nidadas y un

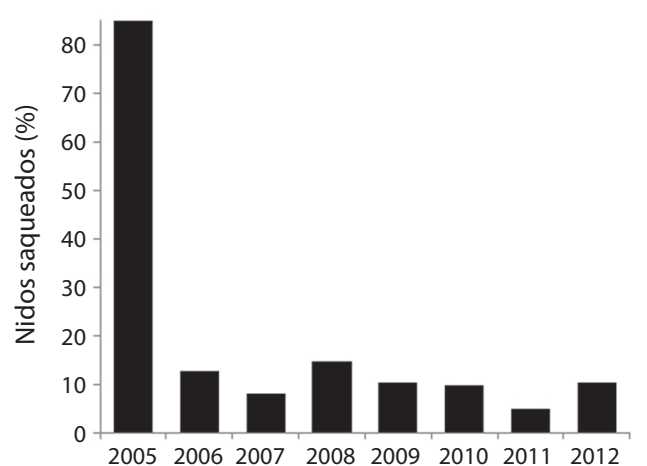

Fig. 5. Porcentaje del número de nidos de L. olivacea saqueados por año, en playa Drake, península de Osa, del 2005 al 2012.

Fig. 5. Incidence of illegal poaching of $L$. olivacea eggs from Drake Beach (2005 to 2012), shown as percentage of total nests laid during each season.

programa de desarrollo y sensibilización comunitaria perfectamente engranado en el proyecto.

Desde 2006, el proyecto ha permitido proteger más del $89 \%$ de los nidos de playa Drake, marcar 335 hembras reproductoras y liberar más de 61000 neonatos al mar. La incidencia del saqueo de huevos se ha mantenido en un promedio del $10.1 \%$, reducción principalmente lograda debido a la vigilancia permanente de nidos reubicados en vivero. Al igual que en muchas otras zonas costeras del Pacífico latinoamericano, el valor cultural del huevo de tortuga como elemento común en la dieta de los pobladores ya sea como fuente proteínica alternativa o como supuesto afrodisiaco, no ha supuesto, sin embargo, una barrera inalcanzable para el desarrollo de iniciativas de conservación. Es más fácil eliminar el hábito, por arraigada que esté, si de ella no depende la sobrevivencia de la población o no supone una actividad muy lucrativa. En este caso, el rápido descenso del saqueo registrado tras la aparición del proyecto de conservación fue favorecido por el débil comercio local existente derivado del huevo de tortuga. Aunque se vendía a pequeña escala, los beneficios económicos derivados de un volumen tan pequeño de nidadas como las registradas en Playa Drake no era lo suficientemente significativo para ejercer 


\section{CUADRO 3}

Valores mínimo y máximo, media y desviación estándar del diámetro y del peso de las muestras con 10 huevos, y de la longitud curva del caparazón (LCC) y ancho curvo del caparazón (ACC) de muestras de 10 neonatos, de los nidos de $L$. olivacea reubicados en vivero, en playa Drake, península de Osa, durante las temporadas 2006-2012

TABLE 3

Minimum and maximum values, mean and standard deviation of the diameter and weight of samples of 10 eggs from each nest relocated to the hatchery, and the curved carapace length (LCC) and width (ACC), and weights of samples of 10 hatchlings from each nest relocated to the hatchery, during each nesting season on Drake Beach from 2006 to 2012

\begin{tabular}{|c|c|c|c|c|c|}
\hline & Valor mínimo & Valor máximo & Media & D.E. & $\mathrm{n}^{*}$ \\
\hline \multicolumn{6}{|c|}{ Temporada 2006} \\
\hline Peso H (g) & 23.0 & 40.0 & 32.3 & 3.05 & n.i. \\
\hline $\mathrm{LCC}(\mathrm{cm})$ & 3.80 & 5.00 & 4.30 & 0.20 & n.i. \\
\hline $\mathrm{ACC}(\mathrm{cm})$ & 4.00 & 5.00 & 4.70 & 1.85 & n.i. \\
\hline Peso C (g) & 11.0 & 23.0 & 18.3 & 2.73 & n.i. \\
\hline \multicolumn{6}{|c|}{ Temporada 2007} \\
\hline Diámetro $(\mathrm{cm})$ & 3.40 & 4.70 & 3.70 & 0.10 & n.i. \\
\hline Peso H (g) & 23.0 & 39.0 & 32.4 & 2.80 & n.i. \\
\hline $\mathrm{LCC}(\mathrm{cm})$ & 3.50 & 4.80 & 4.30 & 0.10 & n.i. \\
\hline $\mathrm{ACC}(\mathrm{cm})$ & 4.00 & 5.30 & 4.50 & 1.10 & n.i. \\
\hline Peso C (g) & 13.0 & 22.0 & 17.7 & 1.80 & n.i. \\
\hline \multicolumn{6}{|c|}{ Temporada 2008} \\
\hline Diámetro $(\mathrm{cm})$ & 3.20 & 4.00 & 3.68 & 0.10 & 47 \\
\hline Peso H (g) & 26.0 & 41.0 & 32.7 & 1.46 & 47 \\
\hline $\mathrm{LCC}(\mathrm{cm})$ & 3.90 & 4.80 & 4.30 & 0.14 & 48 \\
\hline $\mathrm{ACC}(\mathrm{cm})$ & 3.90 & 5.00 & 4.48 & 0.14 & 48 \\
\hline Peso C (g) & 13.0 & 21.0 & 17.2 & 1.21 & 48 \\
\hline \multicolumn{6}{|c|}{ Temporada 2009} \\
\hline Diámetro $(\mathrm{cm})$ & 3.40 & 3.9 & 3.68 & 0.15 & 40 \\
\hline Peso H (g) & 22.8 & 42.2 & 31.0 & 1.29 & 40 \\
\hline $\mathrm{LCC}(\mathrm{cm})$ & 3.81 & 5.45 & 4.44 & 0.15 & 39 \\
\hline $\mathrm{ACC}(\mathrm{cm})$ & 3.81 & 5.45 & 4.49 & 0.14 & 39 \\
\hline Peso C (g) & 14.1 & 20.8 & 17.3 & 0.83 & 39 \\
\hline \multicolumn{6}{|c|}{ Temporada 2010} \\
\hline Diámetro $(\mathrm{cm})$ & 3.15 & 3.99 & 3.58 & 0.11 & 91 \\
\hline Peso H (g) & 24.0 & 39.2 & 33.1 & 1.36 & 91 \\
\hline $\mathrm{LCC}(\mathrm{cm})$ & 4.13 & 4.89 & 4.47 & 0.17 & 49 \\
\hline $\mathrm{ACC}(\mathrm{cm})$ & 4.18 & 4.99 & 4.52 & 0.19 & 49 \\
\hline Peso C (g) & 14.1 & 21.7 & 17.6 & 1.73 & 55 \\
\hline \multicolumn{6}{|c|}{ Temporada 2011} \\
\hline Diámetro $(\mathrm{cm})$ & 3.10 & 3.95 & 3.59 & 0.16 & 101 \\
\hline Peso H (g) & 23.6 & 38.7 & 32.5 & 4.34 & 101 \\
\hline $\mathrm{LCC}(\mathrm{cm})$ & 3.38 & 5.41 & 4.43 & 0.31 & 101 \\
\hline $\mathrm{ACC}(\mathrm{cm})$ & 4.10 & 5.66 & 4.58 & 0.25 & 101 \\
\hline Peso C (g) & 13.2 & 29.0 & 18.6 & 2.78 & 101 \\
\hline \multicolumn{6}{|c|}{ Temporada 2012} \\
\hline Diámetro $(\mathrm{cm})$ & 3.22 & 3.87 & 3.55 & 0.14 & 109 \\
\hline Peso H (g) & 16.6 & 38.4 & 31.4 & 3.90 & 109 \\
\hline $\mathrm{LCC}(\mathrm{cm})$ & 4.02 & 4.98 & 4.44 & 0.18 & 99 \\
\hline $\mathrm{ACC}(\mathrm{cm})$ & 4.02 & 4.95 & 4.53 & 0.18 & 99 \\
\hline Peso C (g) & 12.6 & 21.2 & 17.1 & 1.79 & 99 \\
\hline
\end{tabular}

$\mathrm{n}$ *: Número de muestras (no individuales). n.i.: No hay información; datos fueron perdidos.

$\mathrm{n} *$ : Number of samples (not number of individuals). n.i.: No information available; data were lost. 
una presión que supusiera una amenaza seria al proyecto. Probablemente, la rápida y progresiva aparición de beneficios económicos en diversos miembros de la comunidad derivados del proyecto supuso una presión definitiva contra quienes pudieran tan sólo pensar en boicotear el programa. Con un saqueo de huevos tan intensivo como el manifestado en playa Drake durante las dos últimas décadas, el uso de un vivero se convierte en una herramienta de conservación y recuperación viable y necesaria.

La distribución temporal de anidación de la tortuga lora durante cada temporada en playa Drake indica que los meses de agosto y septiembre presentan mayor anidación. Esto es consistente con la distribución temporal de anidación reportada en otras playas de anidación localizadas en la costa Pacífica de Costa Rica (Valverde et al., 1998; Brenes Arias, 2012; Viejobueno et al., 2012; Bernal Castro et al., 2013). La distribución espacial de anidación en playa Drake revela que la mayor intensidad de anidación fue entre los mojones 15 y 20. Esta zona se caracteriza por contener una barra arenosa y estar próxima a la desembocadura del río Drake apoyando la hipótesis de que la tortuga lora anida selectivamente en zonas de alta turbidez y baja salinidad.

El promedio del éxito de eclosión para los nidos reubicados en vivero fue significativamente mejor que el de los nidos reubicados en playa durante cinco de las siete temporadas, datos que son similares a aquellos reportado por otros proyectos de conservación de tortuga lora en la costa pacífica de Costa Rica (Viejobueno et al., 2012; Bernal Castro et al., 2013).

La tortuga lora es aparentemente capaz de retrasar la anidación hasta que las condiciones ambientales sean favorables (Plotkin, Rostal, Byles, \& Owens, 1997). El comportamiento de anidación de esta especie ha mostrado ser especialmente coordinado durante eventos de arribada (Pritchard, 1969), y existen evidencias que apoyan la influencia de señales ambientales y sociales que marcan el inicio de la anidación (Cornelius \& Robinson, 1985; Mendonça \& Pritchard, 1986, Plotkin, Byles, Rostal, \& Owens, 1995; Plotkin et al., 1997).
Las arribadas de la tortuga lora en el Pacífico oriental coinciden generalmente con el tercer cuarto de luna, y justo antes o durante la marea alta (Plotkin, 1994). Por otro lado, todavía no hay evidencias suficientes para demostrar una coordinación expresa en el proceso de anidación de las tortugas loras en las playas de anidación solitarias y mucho menos las pautas por las que se regiría la misma. En playa Drake, con frecuencia, se registró una ausencia de actividad de anidación en playa sin salidas de tortugas durante varias noches consecutivas, algo que generalmente era seguido por varias salidas simultaneas durante la misma noche y en un rango horario estrecho, un hecho que posiblemente podría interpretarse como un proceso coordinado de anidación. El análisis comparativo entre la frecuencia de anidación y las fases lunares indica que la mayor intensidad de anidación tiene lugar 3-5 días después de la luna nueva y la luna llena. Además se registró la existencia de muy pocas salidas de tortuga durante las mareas altas y una diferencia significativa de actividad a lo largo de la noche con más salidas antes de la medianoche que después. Por lo tanto, se propone que las tortugas loras solitarias que anidan en playa Drake pueden retrasar su anidación hasta que existan las condiciones ambientales favorables, y que los días de mayor intensidad de anidación se caracterizarían por periodos de mareas vivas, con marea baja o marea decreciente y antes de la medianoche. Estos datos indican que la luna podría influir en el comportamiento de anidación solitaria de la tortuga lora, con la marea viva posiblemente sirviendo como un factor de inducción. Además, en base a nuestros hallazgos y las comparaciones con otras playas de anidación recogidas en la literatura, las señales ambientales podrían ser distintas para las tortugas loras en playas de anidación solitarias y las que participan en las arribadas.

Dado que el proyecto lleva operativo solamente siete años, los datos recopilados son aún insuficientes para confirmar una tendencia positiva que muestre una recuperación de la población de tortuga lora que anida en playa Drake. De igual forma, y pese a los excelentes 
logros alcanzados con la significativa reducción del saqueo de nidos, todavía no ha sido neutralizada esta amenaza por completo. Sin protección durante la temporada de anidación, es muy probable que la intensidad del saqueo aumentara, y esta frágil población de tortugas marinas difícilmente sobreviviría por mucho tiempo. Por lo tanto, es muy importante que las actividades de conservación continúen durante futuras temporadas de anidación en bahía Drake al menos hasta que se logre mitigar el consumo local del huevo de tortuga. Tratándose de un hecho fuertemente arraigado culturalmente, serán fundamentales los esfuerzos a nivel de sensibilización ambiental ejercidos sobre la población local. Este proceso, sin duda, puede requerir de una escala intergeneracional donde será preciso que los mecanismos que disparen estos esfuerzos de conservación estén dirigidos desde las comunidades receptoras. No obstante, el saqueo sólo es una de las numerosas amenazas que enfrentan estas criaturas. La pérdida de hábitat de anidación debida al desarrollo costero, la pesca incidental debida a artes poco selectivas como las redes camaroneras o la contaminación del medio marino ejercen una presión mucho más difícil de cuantificar que podría estar acelerando la drástica reducción de la población de tortugas marinas en Playa Drake. Si bien es preciso asegurar la protección de las nidadas en playa, deben impulsarse más acciones en el medio marino donde, no olvidemos, la tortuga pasa la inmensa mayoría de su vida. Se precisa de una inversión en investigación que nos ayude a identificar áreas marinas importantes (corredores, áreas de alimentación y reproducción) que usen frecuentemente las especies de tortugas marinas. Además, el trabajo con las autoridades competentes puede ser clave para ordenar e impulsar medidas que mitiguen la captura incidental en estas áreas críticas identificadas. Debería de impulsarse un plan de acciones integral cuyas medidas de contingencia en una zona no viniera a afectar negativamente a las otras. Este es el ejemplo del movimiento de los barcos camaroneros expulsados del Golfo Dulce hacia la zona de Drake y el humedal Térraba-Sierpe. Creemos firmemente que sin un plan integral de medidas con objetivos de conservación claros, sólo contaremos con parches que lejos de erradir el problema, más bien seguiremos trasladándolo a otras zonas. Hay tecnología simple y económica para reducir el índice de captura accidental de estas y otras especies. Pero hay que tener la voluntad política a nivel internacional para hacerlas efectivas.

\section{AGRADECIMIENTOS}

El financiamiento de este proyecto fue provisto principalmente por Humane Society International, US Department of the Interior, Costa Rican Conservation Fund, Conservation International, y Pronature. Se agradece a Alejandra Monge, Patricio Alonso y Mike Bigler, Miguel Andreu, Alison Le-Garec, Irene Brandts, Kira James, Stephanie Cole, y la comunidad de El Progreso de Drake. Finalmente, agradecemos a los revisores anónimos cuyos comentarios mejoraron este manuscrito.

\section{RESUMEN}

Desde 2006 a 2012, la anidación de la tortuga lora (Lepidochelys olivacea) fue monitoreada en bahía Drake, un enclave reconocido internacionalmente por su excelente oferta eco-turística que se localiza en la región noroeste de la península de Osa. Sorprendentemente, esta área dispone de playas de anidación solitaria de tortuga lora que han permanecido casi desadvertidas hasta fechas recientes. Durante este periodo de monitoreo, 958 nidos fueron registrados en playa Drake (promedio anual: 136.9; densidad: 3.80 nidos/100m de playa), de los cuales 363 (37.9\%) fueron reubicados a un vivero. Antes de 2006, la pérdida anual de nidos fue superior al $85 \%$ debido al saqueo en playa; desde 2006, el porcentaje del saqueo de nidos se mantuvo en un promedio del 10.1\%. Además, un total de 335 hembras fueron identificadas con placas metálicas; el promedio de la longitud curva del caparazón fue de $66.1 \mathrm{~cm}$; el promedio del ancho curvo del caparazón fue de $70.2 \mathrm{~cm}$, y el tamaño promedio por nidada fue de 96.3 huevos. El promedio del éxito de eclosión para los nidos reubicados en vivero fue de $79.2 \%$, y más de 61000 neonatos fueron liberados al mar durante este periodo. Este proyecto es un ejemplo de una iniciativa exitosa de conservación, eco-turismo y desarrollo comunitario.

Palabras clave: Tortuga Lora, Cheloniidae, biología de anidación, tortuga marina, saqueo, conservación comunitaria. 


\section{REFERENCIAS}

Abreu-Grobois, A. \& Plotkin, P. (2008). Lepidochelys olivacea. In IUCN Red List of Threatened Species 2013 (Version 2013.2.). Retrived from http: www. iucnredlist.org

Bernal Castro, M., Sánchez Mendoza, M., Cheetham, S. E., \& Herrera Vargas, H. A. (2013). Conservación de tortugas marinas en playa Piro, playa Pejeperro, y playa Carate, Península de Osa, Costa Rica (Reporte técnico). Puero Jiménez, Costa Rica: Osa Conservación.

Brenes Arias, O. (2012). Conservación de Tortugas Marinas en Reserva Playa Tortuga, Ojochal, Costa Rica (Reporte técnico). Ojachal, Costa Rica: Reserva Playa Tortuga.

Crastz, F. (1982). Embryological stages of the marine turtle Lepidochelys olivacea (Eschscholtz). Revista de Biología Tropical, 30, 113-120.

Cornelius, S. E. \& Robinson, D. C. (1985). Abundance, distribution, and movement of olive ridley sea turtles in Costa Rica (V. Final Report to USFWS). Albuquerque, New Mexico.

Cornelius, S. E., Ulloa, M. A., \& Robinson, D. C. (1991). Management of olive ridley sea turtles (Lepidochelys olivacea) nesting at Playas Nancite and Ostional, Costa Rica. In J. G. Robinson \& K.H. Redford (Eds.), Neotropical wildlife use and conservation (pp. 111-135). Chicago, USA.: The University of Chicago Press.

Drake, D. L. (1996). Marine turtle nesting, nest predation, hatch frequency, and nesting seasonality on the Osa Peninsula, Costa Rica. Chelonian Conservation Biology, 2, 89-92.

Dutton, P. H., Davis, S. K., Guerra, T., \& Owens, D. (1996). Molecular phylogeny for marine turtles based on sequences of the ND4-leucine tRNA and control regions of mitochondrial DNA. Molecular phylogeny for marine turtles based on sequences of the ND4leucine tRNA and control regions of mitochondrial DNA. Molecular Phylogenetics and Evolution, 5(3), 511-21.

Frazier, J. (2003). Prehistoric and ancient historic interactions between humans and marine turtles. In P. L. Lutz, J. A. Musick, \& J. Wyneken (Eds.), Biology of Sea Turtles, Volume II (pp. 1-38). Boca Raton: CRC Press.

Jackson, J. B. C., Kirby, M. X., Berger, W. H., Bjorndal, K. A., Botsford, L. W., Bourque, B. J., ... Warner, R. (2001). Historical overfishing and the recent collapse of coastal ecosystems. Science, 293, 629-638.

James, R., James, K. L., Adams, M., Amo, A., Sullivan, C. E., \& Brandts, I. (2011). Investigation and Conservation of Sea Turtles, Drake Bay, Osa Peninsula,
Costa Rica (Technical Report). San José, Costa Rica: Fundación Corcovado.

James, R., Cole, S. F., White, N., Pita, H., Andrews, G., \& Camacho, L. (2012). Investigation and Conservation of Sea Turtles, Bahía Drake, Osa Peninsula, Costa Rica (Technical Report). San José, Costa Rica: Fundación Corcovado.

James, R., Varela-Murillo, M., Eraso-Rodríguez, S., García-Solà, A., Cooch, D., \& García Cordero, O. (2013). Investigation and Conservation of Sea Turtles, Drake Bay, Osa Peninsula, Costa Rica (Technical Report). San José, Costa Rica: Fundación Corcovado.

Karl, S. H. \& Bowen, B. W. (1999). Evolutionary Significant Units versus Geopolitical Taxonomy: Molecular Systematics of an Endangered Sea Turtle (genus Chelonia). Conservation Biology, 13(5), 990-999.

Le-Garec, A., Melero, D., \& Andreu, M. A. (2010). Community-based development and its application to sea turtle conservation in Bahía Drake, Osa Peninsula, Costa Rica. XXXI Simposio Internacional sobre Conservación y Biología de Tortugas Marinas. Goa, India.

Mendonça, M. T. \& Pritchard, P. (1986). Offshore movements of post nesting Kemp's ridley sea turtles (Lepidochelys kempi). Herpetologica, 42, 373-381.

McCoy, C. J., Vogt, R. C., \& Censky, E. J. (1983). Temperature-controlled sex determination in the sea turtle Lepidochelys olivacea. Journal of Herpetology, 17, 404-406.

Melero, D., Alonso, P., Rubiano, D., \& Careaga, O. (2008). Proyecto de Protección, Conservación y Recuperación de Poblaciones de Tortuga Marina en Playa Drake, Península de Osa-Costa Rica (Reporte técnico). San José, Costa Rica: Fundación Corcovado.

Melero, D., Le-Garec, A., Andreu, M. A., Ramírez, P., \& Moreno, D. (2009). Proyecto de Protección, Conservación y Recuperación de Poblaciones de Tortuga Marina en Playa Drake, Península de Osa -Costa Rica (Reporte técnico). San José, Costa Rica: Fundación Corcovado.

Melero, D., Brandts, I., James, R., Ramírez, P., Hernández, M., \& Sánchez, A. (2010). Proyecto de Protección, Conservación y Recuperación de Poblaciones de Tortuga Marina en Playa Drake, Península de Osa -Costa Rica (Reporte técnico). San José, Costa Rica: Fundación Corcovado.

Merchant-Larios, H., Ruiz-Ramírez, S., Moreno-Mendoza, N., \& Marmolejo-Valencia, A. (1997). Correlation among thermo-sensitive period, estradiol response, and gonad differentiation in the sea turtle Lepidochelys olivacea. General and Comparative Endocrinology, 107, 373-385.

Mortimer, J. A. \& Donnelly, M. (2008). Eretmochelys imbricata. In IUCN Red List of Threatened Species 
2013 (Version 2013.2.). Retrived from http: www. iucnredlist.org

Mrosovsky, N. \& Yntema, C. L. (1980). Temperature dependence of sexual differentiation in sea turtles: Implications for conservation practices. Biological Conservation, 18, 271-280.

Plotkin, P.T. (1994). Migratory and reproductive behavior of the olive ridley turtle Lepidochelys olivacea (Eschscholtz, 1829), in the eastern Pacific Ocean (Doctoral dissertation). Texas A \& M University, College Station, Texas, USA.

Plotkin, P. T., Byles, R. A., Rostal, D. C., \& Owens, D. W. (1995). Independent versus socially facilitated oceanic migrations of the olive ridley, Lepidochelys Olivacea. Marine Biology, 122, 137-143.

Plotkin, P. T., Rostal, D. C., Byles, R. A., \& Owens, D. W. (1997). Reproductive and Developmental Synchrony in Female Lepidochelys olivacea. Journal of Herpetology, 31(1), 17-22.

Pritchard, P. C. G. (1969). Studies of the systematics and reproductive cycles of the genus Lepidochelys (Doctoral dissertation). University of Florida, Gainesville, Florida, USA.

Pritchard, P., Bacon, F. Berry, A. Carr, J. Fletmeyer, R. Gallagher, S., ... Witham, R. (1983). Manual sobre técnicas de investigación y conservación de las tortugas marinas. Washington, D.C., USA: Center for Environmental Education.

Pritchard, P. (2007). Arribadas I Have Known. In P. T. Plotkin (Ed.), Biology and Conservation of Ridley Sea Turtles (pp. 12). Baltimore, Maryland: The John Hopkins University Press.

Sánchez, F., Melero, D., Alonso, P., \& Bigler, M. (2006). Proyecto de Protección, Conservación y Recuperación de Poblaciones de Tortuga Marina en Playa Drake, Península de Osa-Costa Rica (Reporte técnico). San José, Costa Rica: Corcovado Fundation.
Seminoff, J. A. (2004). Chelonia mydas. In IUCN Red List of Threatened Species 2013 (Version 2013.2.). Retrived from http: www.iucnredlist.org

Spotila, J. R., Standora, E. A., Morreale, S. J., \& Ruiz, G. J. (1987). Temperature dependent sex determination in the green turtle (Chelonia mydas): effects on the sex ratio on a natural nesting beach. Herpetologica, 43, 74-81.

Spotila, J. R., Reina, R. D., Steyermark, A. C., Plotkin, P. T., \& Paladino, F. V. (2000). Pacific leatherback turtles face extinction. Nature, 405, 529-530.

Valverde, R. A., Cornelius, S. E., \& Mo, C. L. (1998). Decline of the olive ridley sea turtle (Lepidochelys olivacea) nesting assemblage at Nancite Beach, Santa Rosa National Park, Costa Rica. Chelonian Conservation and Biology, 3, 58-63.

Viejobueno, S., Adams, C. \& Arauz, R. (2012). Conservación e Investigación de Tortugas Marinas en el Pacifico de Costa Rica (Refugio Nacional de Vida Silvestre Caletas-Ario, San Miguel, Costa de Oro y Corozalito) (Reporte técnico). San Francisco de Coyote, Costa Rica: PRETOMA.

Wallace, B. P., DiMatteo, A. D., Bolten, A. B., Chaloupka, M. Y., Hutchinson, B. J., Abreu-Grobois, F. A, ... Mast, R. B. (2011). Global Conservation Priorities for Marine Turtles. PLoS ONE, 6(9).

Wallace, B. P., Tiwari, M., \& Girondot, M. (2013). Dermochelys coriacea. In IUCN Red List of Threatened Species 2013 (Version 2013.2.). Retrived from http: www.iucnredlist.org

Wibbels, T., Rostal, D., \& Byles, R. (1998). High pivotal temperature in the sex determination of the olive ridley sea turtle from Playa Nancite, Costa Rica. Copeia, 1086-1088.

Wibbels, T. (2003). Critical approaches to sex determination in sea turtles. In P. L. Lutz, J. A. Musick, \& J. Wyneken (Ed.), Biology of Sea Turtles, Vol. II. (pp. 103-134). Boca Raton, Costa Rica: CRC Press. 
